\title{
Spectral reflectance and transmittance prediction model for stacked transparency and paper both printed with halftone colors
}

\author{
Jacques Machizaud and Mathieu Hébert* \\ Université de Lyon, Université Jean-Monnet de Saint-Etienne; CNRS, UMR 5516, \\ Laboratoire Hubert Curien, F-4200o, Saint-Etienne, France \\ ${ }^{*}$ Corresponding author: mathieu.hebert@univ-st-etienne.fr
}

Received November 22, 2011; revised May 24, 2012; accepted May 25, 2012; posted May 30, 2012 (Doc. ID 158349); published July 12, 2012

\begin{abstract}
When a transparency printed with a first halftone color is deposited on top of a paper printed with a second halftone color, we obtain a third color that we are able to predict in both reflectance and transmittance modes, thanks to a spectral prediction model. The model accounts for the multiple reflections of light between the printed paper and the printed transparency, which are themselves described by specific reflectance and transmittance models, each one being calibrated using a small number of printed colors. The model can account for light scattering by the inks. The measuring geometry and the orientations of light in the transparency are taken into account on the basis of radiometric rules and geometrical optical laws. Experimental testing carried out from several inkjet-printed CMY halftones shows fairly good agreement between predictions and measurements. (c) 2012 Optical Society of America

OCIS codes: $\quad 100.2810,120.5700,120.7000$.
\end{abstract}

\section{INTRODUCTION}

Thanks to the models developed over the past few decades, the color of halftone prints can be predicted with a satisfactory accuracy for most common printing systems, e.g., offset, inkjet, and electrophotography. The best predictions are provided by spectral models taking into account the spreading of the ink dots on the printing support according to the method introduced by Hersch and Crété [1]. There exist two main families of models, which show similar performance in terms of prediction accuracy: surface models, including the spectral Yule-Nielsen model [2], and the multiple reflection models, including the Clapper-Yule model [3] and the WilliamsClapper model extended to halftones [4,5]. Either type of model is calibrated from the spectral measurement of a few colors printed with the selected printing system, and they are now available in both reflectance and transmittance modes, thanks to the recently proposed extensions of spectral reflectance models to spectral transmittance [ $\underline{5}-\underline{8}]$. In previous works, mathematical methods based on geometrical radiometry were exposed in order to facilitate the derivation of analytical reflectance and transmittance expressions for multilayers composed of layers each one either nonscattering or strongly diffusing, but we did not so far consider halftoned multilayers [9]. We now want to combine the models for halftones and for multilayers. As a first step forward in this direction, our aim in the present work is to predict the spectral reflectance and transmittance of specimens obtained by superposition of a transparency and a diffusing paper, both printed with distinct halftones.

The idea of superposing a transparency and a paper is simple. It may be considered, in reflectance mode, as a special case of the piles of colored nonscattering acetate sheets superposed to a diffusing background, whose study was presented in [10]. Indeed, this previous model and the one that is introduced here lie on similar concepts. However, the fact that the transparency and the paper are printed in halftone raises several difficulties. A first difficulty comes from the fact that the color of the printed transparency is not continuous as for the acetate sheets but composed of a mosaic of colorants resulting from the partial superposition of the ink dots. As many halftone colors may be printed, it would not be reasonable to measure the transparency's spectral reflectance and transmittance for all of them. We therefore need a prediction model adapted to transparencies printed in halftone and calibrated from a small set of printed colors. This model shall take into account the spreading of the inks ("mechanical dot gain") as well as the well-known Yule-Nielsen effect [11], also called "optical dot gain," which occurs when light transits from one colorant in the halftone to another one, either due to scattering in the printed support or, as in the present case, due to multiple reflections between the interfaces. The models for printed transparencies found in the literature $[\underline{12}, 13]$ cope only with the spectral transmittance and do not account for the optical dot gain effect. We show that by taking this effect into account in our model, we improve considerably the prediction accuracy in transmittance mode. An additional difficulty comes from the slight scattering of light by the inks. In paper printing, scattering by the inks is very small compared to the scattering by the paper and can generally be ignored. However, it becomes significant on a specular reflector, such as a transparency. The reflectance prediction model thus needs to be corrected so as to account for that small scattering effect. The last difficulty comes from the moiré phenomena appearing when rotated cluster halftone screens are superposed [14]. Even though perfect registration between 
the halftone screens avoids moirés, the best solution to prevent it is to use a stochastic halftoning method $[\underline{15}, \underline{16}]$.

The model relies on a description of flux transfers between the light sources, the transparency, the paper, and the detector. We consider one light source at each side of the specimen, one for the reflectance mode and one for the transmittance mode. The angular distribution of the incident light is taken into account in the flux transfer model, as well as the geometry of detection. We obtain analytical expressions for the reflectance and transmittance of the specimen as functions of the reflectances and transmittances of the transparency and the paper. In order to predict the reflectance and the transmittance of the printed paper, we may select among several existing models: the spectral Yule-Nielsen model [2] and its extension to transmittance [7], or the Clapper-Yule model [3] or Williams-Clapper model extended to halftones [4] and their respective extensions to transmittance, which are both presented in [8]. Regarding the printed transparency, we introduce an original reflectance and transmittance model, which accounts for the Yule-Nielsen effect and enables including a component in case of significant scattering of light by the inks. The two models for printed transparency and for printed paper are calibrated separately, and no subsequent calibration is needed when paper and transparency are superposed.

In order to explain the physical basis of the model, we first recall useful radiometric rules in Section 2 and optical laws in Section 3 . We also recall in a Section $\underline{4}$ how the spectral reflectance and transmittance of printed papers can be accurately predicted using the Yule-Nielsen modified spectral Neugebauer model accounting for ink-spreading (IS-YNSN model). We present the model for the printed transparency alone in Section $\underline{5}$, then the model for the transparency superposed to the printed paper in Section $\underline{6}$. We discuss the prediction accuracy from several experiments in Section 7 , and we finally draw conclusions.

\section{RADIOMETRIC BACKGROUND}

The color prediction model proposed for a printed transparency on top of a printed paper describes the propagation of light in a stack as a combination of elementary phenomena: reflection and refraction by the interfaces, diffuse reflection by the paper, and spectral attenuation by the inks. The amounts of light concerned by these elementary phenomena are assessed thanks to radiometric concepts [17]. One of the most important laws in radiometry is the Lambert law, which states that when light is perfectly diffused, the radiance is the same in all directions. The radiance $L$, defined as the flux element per unit apparent area and per unit solid angle, and the irradiance $E$, defined as the total flux per unit area, are then related by the following simple equation:

$$
L=E / \pi
$$

The reflectance concept characterizes the ability of surfaces to reemit part of the light they receive. It is defined as the ratio of the reflected flux by a surface element $\Delta S$ and observed through a solid angle $\Gamma_{0}$ to the incident flux on $\Delta S$ coming through a solid angle $\Gamma_{i}$. Each of the two solid angles may be hemispherical $(2 \pi s r)$, conical, or directional (infinitesimal solid angle), thus giving rise to the nine typical $\left(\Gamma_{i}, \Gamma_{0}\right)$ reflectance geometries defined by Nicodemus [18]. Transmit- tance is defined in the same way for light transmitted through the surface. We will be especially interested in the bihemispherical reflectance where $\Gamma_{i}=\Gamma_{0}=2 \pi s r$, the hemispherical-directional reflectance where $\Gamma_{i}=2 \pi \mathrm{sr}$ and $\Gamma_{0}=d \omega_{0}=\sin \theta_{0} d \theta_{0} d \phi_{0}$, and the bidirectional transmittance where both solid angles are infinitesimal. We assume that the incident flux is Lambertian; i.e., the incident radiance is uniform over the solid angle $\Gamma_{0}$, and that the surfaces have homogeneous optical properties in average over areas of a few millimeters. We consider temporally incoherent and unpolarized incident light, also called natural light [19].

In the following experiments, we will use for both reflectance and transmittance measurements the hemisphericaldirectional geometry, where the incident light is Lambertian and the detector captures the directional radiance reflected in one direction (see Fig. 1). We denote as $E_{A}$ and $E_{A}^{\prime}$ the Lambertian incident irradiances in reflectance and transmittance modes, respectively. The radiance directed toward the detector is denoted as $L_{D}$.

The reflectance and the transmittance of the sample with these hemispherical-directional geometries correspond to the following ratios:

$$
\begin{aligned}
& R=\xi \frac{L_{D}}{E_{A}}, \\
& T=\xi \frac{L_{D}}{E_{A}^{\prime}},
\end{aligned}
$$

where $\xi$ is a constant depending only on the detection geometry, whose meaning is explained in Appendix A.

However, a commercial spectrophotometer $\bar{d}$ does not measure directly a reflectance or transmittance but a reflectance factor or transmittance factor in respect to a perfectly white diffuser in reflectance mode and the perfect transmitter which is simply air in transmittance mode. In Appendix A, we derive from radiometric computation the following relations between the reflectance and transmittance factors, the radiance $L_{D}$ and the incident flux $E_{A}$, respectively $E_{A}^{\prime}$ :

$$
\begin{aligned}
& \hat{R}=\pi \frac{L_{D}}{E_{A}}, \\
& \hat{T}=\pi \frac{L_{D}}{E_{A}^{\prime}} .
\end{aligned}
$$

We also show that when the sample is a Lambertian reflector, the reflectance factor and the bihemispherical reflectance are equal.

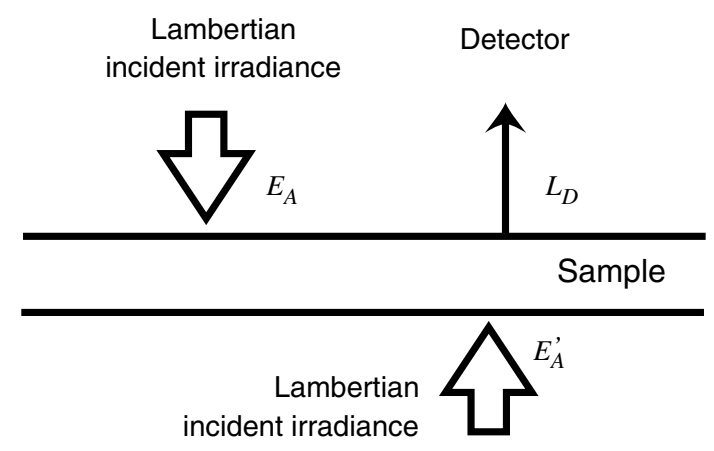

Fig. 1. Measuring geometry in reflectance mode (only $E_{A}$ is received) and transmittance mode (only $E_{A}^{\prime}$ is received). 
In this work, we follow the recommendations by the CIE [20] regarding the measurement geometries. In reflectance mode, we consider a spectrophotometer composed of an integrating sphere, which provides Lambertian illumination and of a detector capturing at $8^{\circ}$ to the normal of the sample $\left(d: 8^{\circ}\right.$ geometry). As the detector is shifted by $8^{\circ}$ from the normal, the light specularly reflected by the surface may be either included in the measurement ( $d i: 8^{\circ}$ geometry) or excluded (de: $8^{\circ}$ geometry). In transmittance mode, the sample is illuminated by Lambertian light from the back side, and the detector is placed in the normal direction at the front side $\left(d: 0^{\circ}\right.$ geometry).

\section{OPTICAL BACKGROUND}

In a nonscattering medium with absorption coefficient $\alpha$, light is exponentially attenuated as a function of the traveled distance $h$. According to Beer's law [21], the ratio of attenuated to incident directional flux is

$$
t=\exp (-\alpha h)
$$

When crossing a layer of this medium with thickness $h$, the flux is attenuated by the same factor $t$, which corresponds to the bidirectional transmittance in the normal direction and will be called normal transmittance. If the directional flux forms an angle $\theta$ in respect to the normal direction, it travels a distance of relative length $h / \cos \theta$ and is therefore attenuated by a factor:

$$
\exp (-\alpha h / \cos \theta)=t^{1 / \cos \theta}
$$

In the present case, inks and plastic transparency are considered as being nonscattering layers. However, because their refractive index, close to 1.5 , is different from that of the surrounding air, light is reflected and refracted at their boundaries. Assuming that the interfaces are flat, reflection and refraction satisfy Snell's laws [19]. The light beam coming from the medium $j$ at angle $\theta_{j}$ is refracted into medium $k$ at the angle $\theta_{k}$ satisfying the following relation:

$$
n_{j} \sin \theta_{j}=n_{k} \sin \theta_{k}
$$

where $n_{j}$ and $n_{k}$ are the refractive indices of media $j$ and $k$. The proportions of reflected and transmitted fluxes are given by the Fresnel formulas [19]. For natural light coming from medium $j$ at the angle $\theta_{j}$, the reflectivity is

$$
\begin{aligned}
R_{j k}\left(\theta_{j}\right)= & \frac{1}{2}\left[\frac{n_{j}\left(n_{k}^{2}-n_{j}^{2} \sin ^{2} \theta_{j}\right)^{\frac{1}{2}}-n_{k}^{2} \cos \theta_{j}}{n_{j}\left(n_{k}^{2}-n_{j}^{2} \sin ^{2} \theta_{j}\right)^{\frac{1}{2}}+n_{k}^{2} \cos \theta_{j}}\right]^{2} \\
& +\frac{1}{2}\left[\frac{\left(n_{k}^{2}-n_{j}^{2} \sin ^{2} \theta_{j}\right)^{\frac{1}{2}}-n_{j} \cos \theta_{j}}{\left(n_{k}^{2}-n_{j}^{2} \sin ^{2} \theta_{j}\right)^{\frac{1}{2}}+n_{j} \cos \theta_{j}}\right]^{2} .
\end{aligned}
$$

The interface has the same reflectivity when the pencil comes from medium $k$ at the angle $\theta_{k}$ linked to $\theta_{j}$ by the refraction law (ㅇ):

$$
R_{k j}\left(\theta_{k}\right)=R_{j k}\left(\theta_{j}\right)
$$

Because the energy is conserved at the interface, the Fresnel transmittivity $T_{j k}\left(\theta_{j}\right)$ is related to the Fresnel reflectivity by

$$
T_{j k}\left(\theta_{j}\right)=1-R_{j k}\left(\theta_{j}\right)
$$

The reciprocity property stated by Eq. (10) also applies to transmittivity.

At normal incidence $\left(\theta_{j}=\theta_{k}=0\right)$, the reflectivity depends only on the refractive indices:

$$
R_{j k}(0)=\left(\frac{n_{k}-n_{j}}{n_{k}+n_{j}}\right)^{2} .
$$

Note that a light beam coming at $8^{\circ}$ to the normal can be considered as normally incident because there is almost no numerical difference between reflectivities at $8^{\circ}$ and $0^{\circ}$.

When the incident light is Lambertian, the reflectance of the interface is obtained by summing up the reflectivities over the hemisphere. Computation detailed in Ref. [22] shows that this bihemispherical reflectance for light coming from medium $j$ is

$$
r_{j k}=\int_{\theta_{j}=0}^{\pi / 2} R_{j k}\left(\theta_{j}\right) \sin 2 \theta_{j} \mathrm{~d} \theta_{j},
$$

and the bihemispherical transmittance is

$$
t_{j k}=\int_{\theta_{j}=0}^{\pi / 2} T_{j k}\left(\theta_{j}\right) \sin 2 \theta_{j} \mathrm{~d} \theta_{j}=1-r_{j k} .
$$

The reciprocity stated for directional light by Eq. (10) is not true for Lambertian illumination. The bihemispherical reflectance $r_{k j}$ and transmittance $t_{k j}$ at the side of medium $k$ are related to those at the side of medium $j$ by the following equations [22]:

$$
\begin{gathered}
t_{k j}=\left(\frac{n_{j}}{n_{k}}\right)^{2} t_{j k}, \\
r_{k j}=1-\left(\frac{n_{j}}{n_{k}}\right)^{2}\left(1-r_{j k}\right) .
\end{gathered}
$$

\section{REFLECTANCE AND TRANSMITTANCE OF A PRINTED PAPER}

A halftone is a mosaic of colored areas resulting from the partial overlap of dot screens of primary inks. The areas with no ink, those with a single ink layer, and those with two or three superposed ink layers are each considered as a distinct "colorant," also called the Neugebauer primary. For three primary inks (e.g., cyan, magenta, and yellow), one obtains a set of eight colorants: white (no ink), cyan alone, magenta alone, yellow alone, red (magenta \& yellow), green (cyan \& yellow), blue (cyan \& magenta), and black (cyan \& magenta \& yellow). In classical clustered-dot or error-diffusion prints, the fractional area occupied by each colorant can be deduced from the surface coverages of the primary inks according to Demichel's equations [23]. These equations are valid in all cases where the ink halftone dots are laid out independently, e.g., in stochastic halftoning, in error diffusion, or in mutually rotated clustered-dot screens. For cyan, magenta, and yellow primary inks with respective surface coverages $c, m$, and $y$, the surface coverages $a_{k}$ of the eight colorants are, respectively, 


$$
\begin{aligned}
a_{w} & =(1-c)(1-m)(1-y) \\
a_{c} & =c(1-m)(1-y) \\
a_{m} & =(1-c) m(1-y) \\
a_{y} & =(1-c)(1-m) y \\
a_{m+y} & =(1-c) m y \\
a_{c+y} & =c(1-m) y \\
a_{c+m} & =c m(1-y) \\
a_{c+m+y} & =c m y .
\end{aligned}
$$

The contribution of the eight colorants to the global reflectance or transmittance of the halftone depends on their respective surface coverages but in a nonlinear manner. This nonlinearity is due to the scattering of light within the paper bulk, which transports light from one colorant to another one. This phenomena, known as the Yule-Nielsen effect $[11,24]$, is amplified by internal reflections of light at the print-air interface. We propose to recall briefly how the reflectance factor of halftone prints can be accurately predicted thanks to the IS-YNSN [1]. This model follows the same approach as Viggiano [2] in order to take into account the Yule-Nielsen effect by raising all reflectance factors (or, accordingly, transmittance factors) to the power $1 / n$. The spectral reflectance factor of the printed paper is thus given by

$$
\hat{R}_{p}(\lambda)=\left[\sum_{k=1}^{8} a_{k} \hat{R}_{k}^{1 / n}(\lambda)\right]^{n},
$$

where $a_{k}$ denotes the surface coverage of the colorant $k, \hat{R}_{k}(\lambda)$ its spectral reflectance factor measured on a large printed patch containing only this colorant (solid colorant patch) and $n$ a constant to be fitted, called the "Yule-Nielsen factor."

In a recent work [7], the authors showed that this model can be transposed to transmittance in a straightforward manner, from the measured spectral transmittance factors $\hat{T}_{k}(\lambda)$ of the solid colorant patches:

$$
\hat{T}_{p}(\lambda)=\left[\sum_{k=1}^{8} a_{k} \hat{T}_{k}^{1 / n}(\lambda)\right]^{n} .
$$

As inks are liquid, they are absorbed by capillarity by the paper bulk and spread over the other inks. Their effective surface coverages $a^{\prime}$ are higher than the nominal ones. An inkspreading curve provides the relation between nominal and effective surface coverages for one ink superposed with a solid layer of any other ink(s). When one halftoned ink $i$ is superposed with a solid layer of colorant $j$, we have two colorants in the resulting halftone: the colorant $j$ of surface coverage $\left(1-a_{i / j}\right)$, and the second colorant resulting from the superposition of ink $i$ and colorant $j$ of nominal surface coverage $a_{i / j}$. For this halftone, the reflectance factor expression given in Eq. (18) becomes

$$
\hat{R}_{p}^{(i / j)}\left(a_{i / j}, \lambda\right)=\left[\left(1-a_{i / j}\right) \hat{R}_{j}^{1 / n}(\lambda)+a_{i / j} \hat{R}_{i / j}^{1 / n}(\lambda)\right]^{n} .
$$

The effective surface coverage $a_{i / j}^{\prime}$ is fitted so as to minimize the sum of squared differences between the predicted spectral reflectance factor $\hat{R}_{p}^{(i / j)}(\lambda)$ and the measured spectral reflectance factor $\hat{P}_{p}^{(i / j)}(\lambda)$ :

$$
a_{i / j}^{\prime}=\underset{0<a^{\prime}<1}{\arg \min } \sum_{\lambda}\left[\hat{R}_{p}^{(i / j)}\left(a^{\prime}, \lambda\right)-\hat{P}_{p}^{(i / j)}(\lambda)\right]^{2} .
$$

In transmittance mode, the predicted spectral transmittance factor $\hat{T}_{p}^{(i / j)}(\lambda)$ is given by

$$
\hat{T}_{p}^{(i / j)}\left(a_{i / j}, \lambda\right)=\left[\left(1-a_{i / j}\right) \hat{T}_{j}^{1 / n}(\lambda)+a_{i / j} \hat{T}_{i / j}^{1 / n}(\lambda)\right]^{n}
$$

The effective surface coverage $a_{i / j}^{\prime}$ is also fitted so as to minimize the sum of squared differences between the predicted spectral transmittance factor $\hat{T}_{p}^{i / j}(\lambda)$ and the measured spectral transmittance factor $\hat{Q}_{p}^{i / j}(\lambda)$ :

$$
a_{i / j}^{\prime}=\underset{0<a^{\prime}<1}{\arg \min } \sum_{\lambda}\left[\hat{T}_{p}^{(i / j)}\left(a^{\prime}, \lambda\right)-\hat{Q}_{p}^{(i / j)}(\lambda)\right]^{2} .
$$

The $a_{i / j}^{\prime}$ values provided by Eqs. (21) and (23) should be identical for a similar halftone sample. However, the experience shows that they are often slightly different. As shown in [7], the ink-spreading curves partly compensate optical phenomena ignored or misestimated by the model.

In order to calibrate the ink-spreading model, we print each ink at three different nominal surface coverages $a$, typically $0.25,0.5$, and 0.75 , (i) alone on paper, (ii) superposed to a solid layer of the second ink, (iii) superposed to a solid layer of the third ink, and (iv) superposed to a solid layer of the second and the third inks. For the halftone of ink $i$ superposed to the solid colorant $j$ with the nominal surface coverage $a_{i / j}$, we deduce the effective surface coverage $a_{i / j}^{\prime}$ using Eq. (21) in reflectance mode or Eq. (23) in transmittance mode. By linear interpolation between the computed points $\left(a_{i / j}, a_{i / j}^{\prime}\right)$, we obtain the so-called ink-spreading curves $f_{i / j}$ giving the relations $a_{i / j}^{\prime}=f_{i / j}\left(a_{i / j}\right)$ for every nominal surface coverage of ink $i$ on top of the colorant $j$ (see Fig. 2). Linear interpolation seems to be sufficient to get good prediction accuracy. Note however that quadratic interpolation exists, which enables in this case reducing the number of measurements (only halftones printed at 0.5 nominal surface coverage are needed) while providing similar prediction accuracy [25]. For a three-ink printer, each ink can be superposed to four different colorants, which yields 12 ink-spreading curves. As three nominal surface coverages are used to establish each ink-spreading curve, 36 printed colors need to be measured to calibrate the model. The disposition order of the ink, generally unknown with desktop printers, has no importance in this model.

Let us now predict the spectral reflectance of a given halftone with nominal ink surface coverages $c_{0}, m_{0}$, and $y_{0}$. We first compute the effective surface coverages $c, m, y$ of the inks by performing a few iterations of the following equations [1]:

$$
\begin{aligned}
c= & (1-m)(1-y) f_{c / w}\left(c_{0}\right)+m(1-y) f_{c / m}\left(c_{0}\right) \\
& +(1-m) y f_{c / y}\left(c_{0}\right)+m y f_{c /(m+y)}\left(c_{0}\right) \\
m= & (1-c)(1-y) f_{m / w}\left(m_{0}\right)+c(1-y) f_{m / c}\left(m_{0}\right) \\
& +(1-c) y f_{m / y}\left(m_{0}\right)+c y f_{m /(c+y)}\left(m_{0}\right) \\
y= & (1-m)(1-c) f_{y / w}\left(y_{0}\right)+m(1-c) f_{y / m}\left(y_{0}\right) \\
& +(1-m) c f_{y / c}\left(y_{0}\right)+m c f_{y /(m+c)}\left(y_{0}\right) .
\end{aligned}
$$




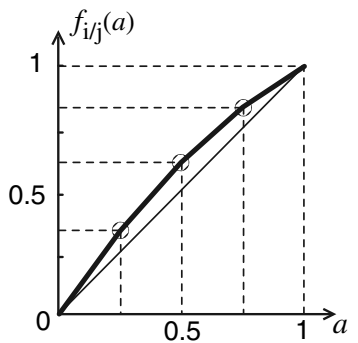

Fig. 2. Example of ink-spreading curve, giving the effective surface coverage of ink $i$ when superposed on colorant $j$ as a function of the nominal surface coverage $a$.

For the first iteration, $c=c_{0}, m=m_{0}$, and $y=y_{0}$ are taken as initial values on the right side of the equations. The obtained values of $c, m$, and $y$ are then inserted again into the right side of the equations, which gives new values of $c, m$, and $y$, and so on, until the values of $c, m, y$ stabilize. Then, the obtained effective surface coverages $c, m$, and $y$ are plugged into the Demichel's equations [see Eq. (17)], which provides the effective surface coverages $a_{k}$ of the eight colorants. Finally, the reflectance and transmittance factors are predicted thanks to Eqs. (18), (19), respectively.

\section{REFLECTANCE AND TRANSMITTANCE OF A PRINTED TRANSPARENCY}

The optical properties of a printed transparency are very different from the ones of a printed paper due to the absence of scattering by the printing support. We introduce an original spectral reflectance and transmittance prediction model inspired of a model previously introduced for uniformly colored films [10]. Let us recall briefly this latter before extending it to halftones.

A nonscattering transparency is composed of two flat interfaces bounding the nonscattering medium, whose refractive index $n_{1}$ is different from the one of the surrounding air (medium 0 , see Fig. 3). The relationship between the light propagation angles in media 0 and 1 , denoted, respectively, as $\theta_{0}$ and $\theta_{1}$, is

$$
n_{0} \sin \theta_{0}=n_{1} \sin \theta_{1} .
$$

A collimated incident light undergoes multiple reflections between the two interfaces by following the directions represented in Fig. 3. In the present case, the transparency thickness is much larger than the coherence length of the everyday light source spectra. There is therefore no significant interference effect. If the transparency was made of glass, the parallel and the perpendicular polarizations would undergo the same trajectories but with different reflectivities. However, on a numerical point of view, taking into account polarization does not bring much to the prediction accuracy. We will therefore assume that light is temporally incoherent and unpolarized at each reflection or transmission.

As the light travels across the plastic layer, it is attenuated by a factor $t_{t}^{1 / \cos \theta_{1}}(\lambda)$, where $t_{t}(\lambda)$ denotes the normal transmittance of the transparency and $\theta_{1}$ its orientation in the transparency. Because $\theta_{1}=\arcsin \left(\sin \theta_{0} n_{1}\right)$, we have

$$
\frac{1}{\cos \theta_{1}}=\left(1-\frac{\sin ^{2} \theta_{0}}{n_{1}^{2}}\right)^{1 / 2} .
$$

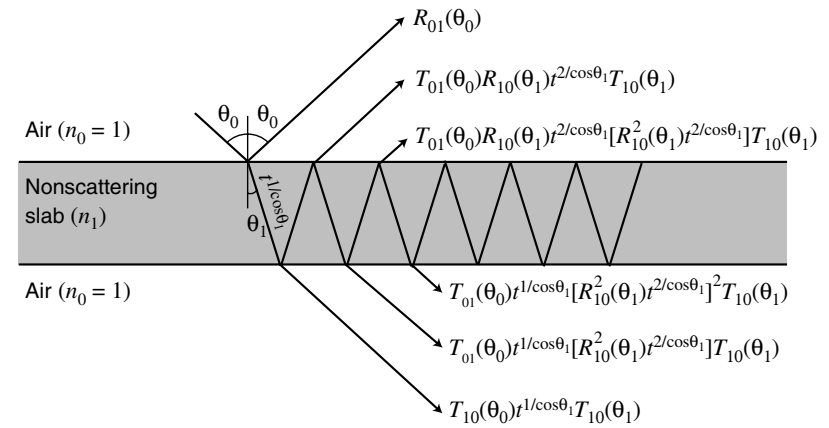

Fig. 3. Reflection and transmission of directional light by a nonscattering film.

By summing on an intensity basis the different components exiting the transparency at each side, one obtains a geometrical series expressing the spectral reflectance $R_{010}\left(\theta_{0}, t_{t}, \lambda\right)$ and transmittance $T_{010}\left(\theta_{0}, t_{t}, \lambda\right)$ for the considered incident angle $\theta_{0}$ :

$$
\begin{gathered}
R_{010}\left(\theta_{0}, t_{t}, \lambda\right)=R_{01}\left(\theta_{0}\right)+\frac{T_{01}^{2}\left(\theta_{0}\right) R_{01}\left(\theta_{0}\right) t_{t}^{2 / \cos \theta_{1}}(\lambda)}{1-R_{01}^{2}\left(\theta_{0}\right) t_{t}^{2 / \cos \theta_{1}}(\lambda)}, \\
T_{010}\left(\theta_{0}, t_{t}, \lambda\right)=\frac{T_{01}^{2}\left(\theta_{0}\right) t_{t}^{1 / \cos \theta_{1}}(\lambda)}{1-R_{01}^{2}\left(\theta_{0}\right) t_{t}^{2 / \cos \theta_{1}}(\lambda)} .
\end{gathered}
$$

If the transparency is coated with a solid colorant layer, Eqs. (27) and (28) remain valid by replacing the normal transmittance $t_{t}(\lambda)$ of the bare transparency with the normal transmittance $t_{k}(\lambda)$ of the transparency coated with colorant $k$.

At normal incidence $\left(\theta_{0}=0^{\circ}\right)$, we have

$$
\begin{gathered}
R_{01}(0)=r_{0}=\left(\frac{n_{1}-n_{0}}{n_{1}+n_{0}}\right)^{2}, \\
T_{01}(0)=1-r_{0} .
\end{gathered}
$$

The reflectance of the printed transparency given by Eq. (27) thus becomes

$$
R_{010}\left(0, t_{k}, \lambda\right)=r_{0}+\frac{\left(1-r_{0}\right)^{2} r_{0} t_{k}^{2}(\lambda)}{1-r_{0}^{2} t_{k}^{2}(\lambda)},
$$

and its transmittance given by Eq. (28) becomes

$$
T_{010}\left(0, t_{k}, \lambda\right)=\frac{\left(1-r_{0}\right)^{2} t_{k}(\lambda)}{1-r_{0}^{2} t_{k}^{2}(\lambda)}
$$

Because the transparency is nonscattering, a detector located in the normal to the transparency collects only the light incoming from the normal direction. $R_{010}\left(0, t_{k}, \lambda\right)$ and $T_{010}\left(0, t_{k}, \lambda\right)$ can be therefore measured with the $d: 0^{\circ}$ geometry that we have selected. The normal transmittance $t_{k}(\lambda)$ of the colorants can be deduced from either one, but because the reflectance of the transparency is very low (at most 0.1 ), the measurement is more subject to noise and therefore less accurate. We thus prefer deducing $t_{k}$ for each wavelength from 
the measured spectral transmittance $T_{010}^{(k)}(\lambda)$, thanks to the following formula derived from Eq. (32):

$$
t_{k}=\frac{\left[\left(1-r_{0}\right)^{4}+4 r_{0}^{2}\left(T_{010}^{(k)}\right)^{2}\right]^{1 / 2}-\left(1-r_{0}\right)^{2}}{2 r_{0}^{2} T_{010}^{(k)}} .
$$

When the transparency is coated with a halftone color, Eqs. (27) and (28) are extended in such a manner as to account for the contribution of the eight colorants in respect to their respective surface coverages $a_{k}$. We assume in a first approach that the halftone is a juxtaposition of small pieces of transparencies coated with different colorants, whose respective areas are larger than significant lateral propagation of light due to multiple reflections between the interfaces (see Fig. 4). In this case, each colorant piece has the same reflectance as the large colorant patch, and the halftone reflectance is simply the mean of these colorant patch reflectances $R_{010}\left(\theta_{0}, t_{k}, \lambda\right)$ in respect to their respective surface coverage $a_{k}$ :

$$
\begin{aligned}
& R_{010}\left(\theta_{0}, a_{k}, t_{k}, \lambda\right)=\sum_{k=1}^{8} a_{k} R_{010}\left(\theta_{0}, t_{k}, \lambda\right), \\
& T_{010}\left(\theta_{0}, a_{k}, t_{k}, \lambda\right)=\sum_{k=1}^{8} a_{k} T_{010}\left(\theta_{0}, t_{k}, \lambda\right) .
\end{aligned}
$$

However, we observe experimentally, as will be discussed in Section 7 from results presented in Table 2, that this linear model is not optimal. Colorant areas are probably not large enough to fully satisfy the assumption made above: light can partly transit from one colorant to another one during the multiple reflections process. These transitions, well known on paper due to the scattering of light in the paper bulk, lead to a similar effect as the Yule-Nielsen effect. We take it into account by applying to Eqs. (34) and (35) the Yule-Nielsen transform, i.e.,

$$
R_{010}\left(\theta_{0}, a_{k}, t_{k}, \lambda\right)=\left[\sum_{k=1}^{8} a_{k} R_{010}^{1 / n}\left(\theta_{0}, t_{k}, \lambda\right)\right]^{n} .
$$

The equivalent for the transmittance is

$$
T_{010}\left(\theta_{0}, a_{k}, t_{k}, \lambda\right)=\left[\sum_{k=1}^{8} a_{k} T_{010}^{1 / n}\left(\theta_{0}, t_{k}, \lambda\right)\right]^{n},
$$

where $n$ is a factor that will be fitted in the calibration step. The improvement of prediction accuracy owing to the YuleNielsen transform is shown in Section 7, Table 2.

Up to now, we have assumed that the transparency and the inks are nonscattering. In practice, however, slight scattering

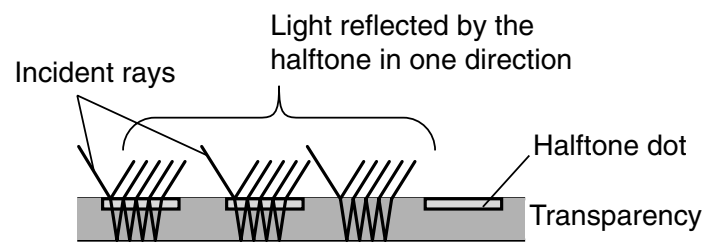

Fig. 4. Light is multiply reflected within each halftone dot. may occur in the inks, yielding a well noticeable effect in reflectance mode. We observe in Fig. 5 that the reflectance of printed transparencies is higher than the reflectance of the unprinted transparency, whereas it would be lower if the inks were purely absorbing. In order to cope with light scattering by the inks, we introduce a Lambertian reflectance component, denoted as $\rho$. For the purpose of comparison, we formally multiply it by a factor $K$ which is 1 when the scattering must be taken into account, or it is 0 when it can be ignored. We assume that the diffuse and directional reflectances are independent of each other (i.e., on average they do not interfere).

Let us consider that the transparency is illuminated by a Lambertian irradiance $E_{A}$, which corresponds to the geometry that we use. The nonscattering part of the transparency and its Lambertian part respond differently to this Lambertian illumination. A fraction $K \rho(\lambda)$ of it is diffused and the radiance $K \rho(\lambda) E_{A} / \pi$ flows toward the detector. The radiance $R_{010}\left(0, a_{k}, t_{k}, \lambda\right) E_{A} / \pi$ reflected by the transparency also flows toward the detector. By dividing the total radiance reflected toward the detector by the incident irradiance $E_{A}$, and multiplying it by $\pi$ (see Appendix A), one obtains the reflectance factor of the transparency:

$$
\hat{R}(\lambda)=R_{010}\left(0, a_{k}, t_{k}, \lambda\right)+K \rho(\lambda) .
$$

By discarding the specular component from measurement, i.e., by using a $d e: 8^{\circ}$ geometry, we measure directly the diffuse reflection component $K \rho(\lambda)$. This obviously makes sense only if the inks are diffusing, i.e., if $K=1$. In the case of a halftone, we can predict the diffuse component $\rho(\lambda)$ from the components $\rho_{k}(\lambda)$ measured on solid layers of the eight colorants:

$$
\rho(\lambda)=\sum_{k=1}^{8} a_{k} \rho_{k}(\lambda)
$$

Equation (39) implicitly assumes that the contribution of each colorant to the diffuse reflection is proportional to its surface coverage. We also assume that the reflectance of the transparency is the same on its two sides. The Helmholtz reciprocity principle states that the reflectance values corresponding to the $0^{\circ}: d$ and $d: 0^{\circ}$ geometries are equal [26].

The calibration of the model consists in determining by measurement the parameters $a_{k}, b_{k}, t_{k}(\lambda), \rho_{k}(\lambda)$. We first want to obtain the normal transmittances $t_{k}(\lambda)$ and the diffuse

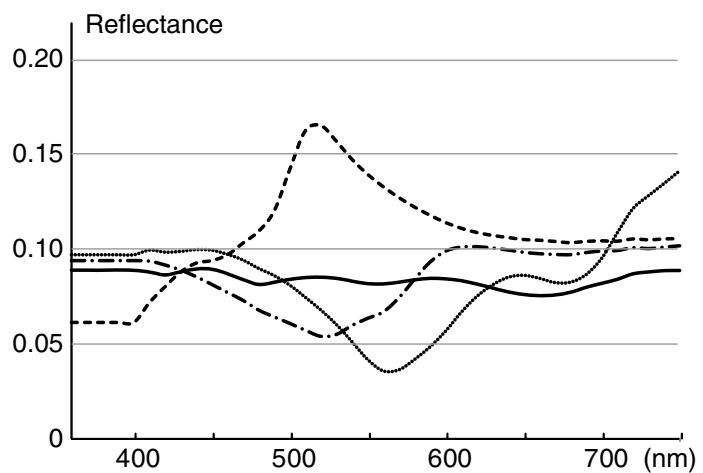

Fig. 5. Spectral reflectance of solid ink layers printed on a transparency: cyan (dotted curve), magenta (dashed-dotted curve), yellow (dashed curve) and no ink (solid curve). 


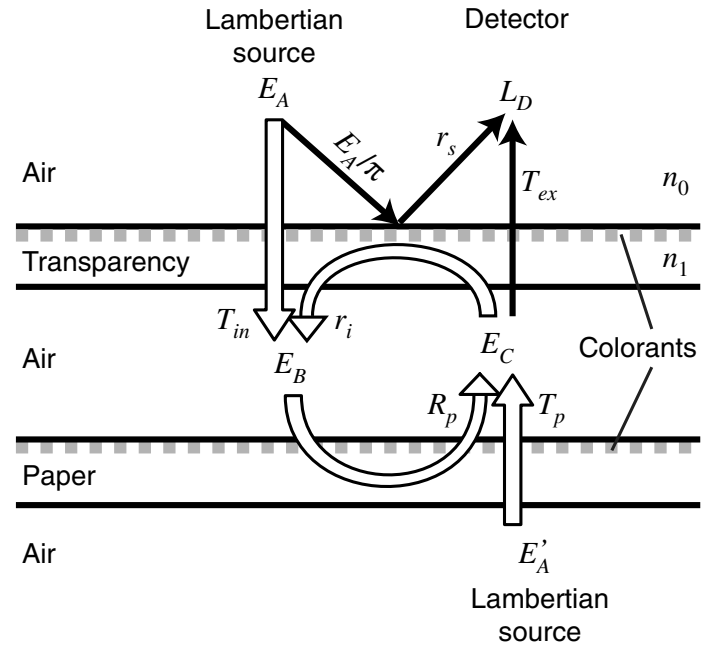

Fig. 6. Reflection and transmission of light by a printed transparency superposed with a printed paper.

reflectances $\rho_{k}(\lambda)$ of the eight colorants. We print solid layers of these colorants on the transparency and measure their transmittance $Q_{t}^{(k)}(\lambda)$ at $0^{\circ}$, as well as their reflectance factor $\hat{P}_{t}^{(k)}(\lambda)$ at $8^{\circ}$ with the specular component excluded. In reflectance mode, the measurements performed on solid colorant patches directly provide the diffuse reflectances $\rho_{k}(\lambda)$. Their measurements in transmittance mode provide the normal transmittances thanks to Eq. (33). Then, we calculate inkspreading curves as for halftones printed on paper. For that purpose, we print 36 halftone patches where one ink is printed at nominal surface coverage $0.25,0.5$, or 0.75 , and the other two inks are printed at nominal surface coverage 0 or 1 . We measure the transmittance of these patches at $0^{\circ}$, whose corresponding expression is

$$
\begin{aligned}
T_{t}^{(i / j)}\left(a_{i / j}, \lambda\right)= & \left\{\left(1-a_{i / j}\right)\left[\frac{\left(1-r_{0}\right)^{2} t_{i}(\lambda)}{1-r_{0}^{2} t_{i}^{2}(\lambda)}\right]^{1 / n}\right. \\
& \left.+a_{i / j}\left[\frac{\left(1-r_{0}\right)^{2} t_{j}(\lambda)}{1-r_{0}^{2} t_{j}^{2}(\lambda)}\right]^{1 / n}\right\}^{n},
\end{aligned}
$$

where $t_{i}(\lambda)$ and $t_{j}(\lambda)$ denote the normal transmittances of the colorants, respectively outside and inside the halftone dots, and $a_{i / j}$ denotes the effective surface coverage of the halftoned ink; $a_{i / j}$ is fitted in such manner as to minimize the sum of squared differences between the spectral transmittance predicted by Eq. (40) and the measured one, $Q_{t}^{(i / j)}(\lambda)$ :

$$
a_{i / j}^{\prime}=\underset{a^{\prime}}{\arg \min } \sum_{\lambda}\left[T_{t}^{(i / j)}\left(a^{\prime}, \lambda\right)-Q_{t}^{(i / j)}(\lambda)\right]^{2} .
$$

The effective surface coverage is calculated for the 36 halftone patches. As it is theoretically independent of the measuring geometry, the same value should be deduced from the measurement in reflectance mode. However, we observe that the reflectance and transmittance modes provide different effective surface coverages, a problem which has already been noticed for halftones printed on paper (see also [7]). This is probably due to the fact that these effective surface coverages take into account optical phenomena ignored in our model. Let us denote as $b_{i / j}^{\prime}$ the effective surface coverages fitted from the measured reflectance factor $\hat{P}_{t}^{(i / j)}(\lambda)$ when the specular reflection is discarded. They are obtained by performing the following minimization:

$$
b_{i / j}^{\prime}=\underset{b^{\prime}}{\arg \min } \sum_{\lambda}\left[\left(1-b^{\prime}\right) \rho_{i}(\lambda)+b^{\prime} \rho_{j}(\lambda)-\hat{P}_{t}^{(i / j)}(\lambda)\right]^{2},
$$

where $\rho_{i}(\lambda)$ and $\rho_{j}(\lambda)$ denote the diffuse reflectances of the colorants respectively outside and inside the halftones dots of the effective surface coverage $b^{\prime}$.

From the 36 calculated values of $a_{i / j}^{\prime}$ and the 36 calculated values of $b_{i / j}^{\prime}$, we establish the ink-spreading curves $f_{i / j}(a)$, respectively $g_{i / j}(b)$, by linear interpolation as in the model for printed paper. Then, for any halftone, we calculate the effective surface coverages $\left\{a_{c}^{\prime}, a_{m}^{\prime}, a_{y}^{\prime}\right\}$ and $\left\{b_{c}^{\prime}, b_{m}^{\prime}, b_{y}^{\prime}\right\}$ of the three inks by iterating formulas (24), then the effective surface coverages $a_{k}^{\prime}$ and $b_{k}^{\prime}$ of the colorants using Demichel's equations (17). Finally, the reflectance factor of the printed transparency illuminated at angle $\theta_{0}$ is

$$
\begin{aligned}
\hat{R}\left(\theta_{0}, a_{k}^{\prime}, t_{k}, b_{k}^{\prime}, \rho_{k}, \lambda, K\right)= & {\left[\sum_{k=1}^{8} a_{k}^{\prime} R_{010}^{1 / n}\left(\theta_{0}, t_{k}, \lambda\right)\right]^{n} } \\
& +K \sum_{k=1}^{8} b_{k}^{\prime} \rho_{k}(\lambda) .
\end{aligned}
$$

The transmittance at angle $\theta_{0}$ is

$$
T\left(\theta_{0}, a_{k}^{\prime}, t_{k}, \lambda\right)=\left[\sum_{k=1}^{8} a_{k}^{\prime} T_{010}^{1 / n}\left(\theta_{0}, t_{k}, \lambda\right)\right]^{n} .
$$

The bihemispherical reflectance for Lambertian incident light is given by

$$
r\left(a_{k}^{\prime}, t_{k}, b_{k}^{\prime}, \rho_{k}, \lambda, K\right)=\int_{\theta_{0}=0}^{\pi / 2} \hat{R}\left(\theta_{0}, a_{k}^{\prime}, t_{k}, b_{k}^{\prime}, \rho_{k}, \lambda, K\right) \sin 2 \theta_{0} \mathrm{~d} \theta_{0},
$$

and the bihemispherical transmittance is given by

$$
t\left(a_{k}^{\prime}, t_{k}, \lambda\right)=\int_{\theta_{0}=0}^{\pi / 2} T\left(\theta_{0}, a_{k}^{\prime}, t_{k}, \lambda\right) \sin 2 \theta_{0} \mathrm{~d} \theta_{0} .
$$

\section{PRINTED TRANSPARENCY ON TOP OF PRINTED PAPER}

In this section, we propose to superpose the transparency and the paper both printed with different halftones. We want to predict the spectral reflectance and transmittance factors of this specimen, knowing the spectral reflectance and transmittance of the transparency at $0^{\circ}$, and the spectral reflectance and transmittance of the printed paper for the $d: 0^{\circ}$ geometry. The printed paper is assumed to be a Lambertian reflector, which is nearly the case of the matte papers. Thus, its reflectance factor, which can be measured or predicted by the model presented in Section 4, is equal to its bihemispherical reflectance. The same remark applies to transmittance. Let us show how the individual reflectances and transmittances of the two prints are combined according to the flux transfers taking place between them.

First of all, we observe that when a transparency is deposited on a paper, there remains a layer of air between them. 
This air slice may be removed by pasting the transparency and the paper with a liquid with similar refractive index as the two substrates, for example oil, whose effect is to cancel the effect of the interfaces (the bottom surface of the transparency and the top surface of the paper) and create optical contact between them. Strong color variation may be observed compared to the case where the air slice is maintained, as shown in the case of plastic sheets in [10]. However, as in the case of printed surfaces, liquid would dilute the inks, we prefer considering the case where an air slice exists.

The incident irradiances $E_{A}^{\prime}$ and $E_{A}$ emitted by the sources are Lambertian and illuminate, respectively, the paper at the back side, and the transparency at the top side (see Fig. 6). In our experiments, the inks are always deposited on top of the transparency and on top of the paper, i.e., in front of the detector. At the top side, a directional flux $L_{D}$ flows toward the detector. A fraction of the incoming irradiance $E_{A}$ is reflected by the transparency and contributes to $L_{D}$. This fraction is denoted as $r_{s}$. A fraction $T_{\text {in }}$ of $E_{A}$ is transmitted through the transparency, including the attenuations by the colorants and by the transparency, and contributes to the irradiance $E_{B}$. This irradiance is then reflected (reflectance $R_{p}$ ) by the printed paper and contributes to the irradiance $E_{C}$. From the back side, the Lambertian irradiance $E_{A}^{\prime}$ is attenuated by the transmittance $T_{p}$ comprising the attenuations by the colorants and the paper bulk and contributes to the irradiance $E_{C}$. While a fraction $T_{\text {ex }}$ of the irradiance $E_{C}$ is transmitted through the transparency toward the detector, another fraction $r_{i}$ is reflected by the transparency and contributes to the irradiance $E_{B}$. One obtains a system of three equations valid for every wavelength:

$$
\begin{aligned}
& E_{B}=T_{\mathrm{in}} E_{A}+r_{i} E_{C} \\
& E_{C}=T_{p} E_{A}^{\prime}+R_{p} E_{B} \\
& L_{D}=r_{s} E_{A}+T_{\mathrm{ex}} E_{C} .
\end{aligned}
$$

When $E_{A}^{\prime}=0$, the fraction $\pi L_{D} / E_{A}$ corresponds to the reflectance factor of the specimen, denoted as $\hat{R}_{t+p}$. When $E_{A}=0$, the fraction $\pi L_{D} / E_{A}^{\prime}$ corresponds to its transmittance factor, denoted as $T_{t+p}$. From Eqs. (47), one deduces

$$
\begin{gathered}
\hat{R}_{t+p}=\pi r_{s}+\pi \frac{T_{\mathrm{in}} T_{\mathrm{ex}} R_{p}}{1-r_{i} R_{p}}, \\
\hat{T}_{t+p}=\pi \frac{T_{p} T_{\mathrm{ex}}}{1-r_{i} R_{p}} .
\end{gathered}
$$

We can predict the reflectance in or out of the specular direction. The difference between these two configurations is simply the inclusion, respectively exclusion, of the reflection component $r_{s}$.

The terms $T_{\mathrm{in}}, T_{\mathrm{ex}}, r_{i}$, and $r_{s}$ are relative to the transparency, while $T_{p}$ and $R_{p}$ are related to the paper. These terms are all wavelength dependent, but for the sake of simplicity in the notation, we remove the term $\lambda$. Let us explain these six terms, assuming that the two supports are coated with different halftone colors. The indices $k$ and $j$ will refer to the colorants printed on the transparency, respectively, on the paper.

$T_{\text {ex }}$ corresponds to the fraction of Lambertian irradiance $E_{C}$, which is directed toward the detector at $0^{\circ}$, thereby to the radiance $E_{c} / \pi$ attenuated according to the transmittance of the printed transparency given by Eq. (44):

$$
T_{\mathrm{ex}}=\frac{1}{\pi} T\left(0, a_{k}^{\prime}, t_{k}, \lambda\right)
$$

$T_{\text {in }}$ corresponds to the fraction of the incident flux $E_{A}$, which is transmitted to the paper bulk. It corresponds to the bihemispherical transmittance given by Eq. (하).

The factor $r_{s}$ corresponds to the reflectance of the transparency. It has a similar expression as the reflectance factor given by Eq. (43) multiplied by a factor $1 / \pi$ :

$$
r_{s}=\frac{1}{\pi}\left[\sum_{k=1}^{8} a_{k}^{\prime} R_{010}^{1 / n}\left(\theta_{0}, t_{k}, \lambda\right)\right]^{n}+\frac{K}{\pi} \sum_{k=1}^{8} b_{k}^{\prime} \rho_{k}(\lambda) .
$$

When the specular component is excluded, the directional component of the reflectance is removed:

$$
r_{s}=\frac{K}{\pi} \sum_{k=1}^{8} b_{k}^{\prime} \rho_{k}(\lambda)
$$

The factor $r_{i}$ corresponds to the fraction of Lambertian flux $E_{C}$, which is internally reflected by the transparency. It therefore corresponds to the bihemispherical reflectance $r_{010}\left(a_{k}^{\prime}, t_{k}, b_{k}^{\prime}, \rho_{k}, \lambda, K\right)$ expressed in Eq. (45). The reflectance of the paper $R_{p}$ is predicted according to the ink-spreading enhanced Yule-Nielsen modified spectral Neugebauer model, i.e., Eq. (18). The transmittance $T_{p}$ is predicted thanks to Eq. (19).

Note that the factor $\pi$ appearing in Eqs. (48) and (49) and the factor $1 / \pi$ appearing in Eqs. (50) and (51) or (52) mutually cancel.

\section{EXPERIMENTAL TESTS}

The model introduced in this paper was tested on different supports printed in inkjet. By printing each of the three inks (cyan, yellow and magenta) at nominal surface coverages $0,0.25,0.5,0.75$, or 1 , we obtained a set of 125 colors that were printed on both the transparency and the paper. Then, by superposition of the two prints, we obtained $125 \times 125$ possible halftone combinations, among which 100 samples were selected and measured in reflectance and transmittance modes with the X-Rite Color i7 instrument. In order to assess the influence of the moiré phenomenon, we tested two halftoning methods. In the first case, we used stochastic halftoning in order to prevent moiré $[15,16]$. In the second case, we used classical rotated cluster halftoning of same period for the two prints. Moiré patterns occur when they are superposed, but we can make it invisible by aligning them as perfectly as possible, the moiré period becoming much larger than the size of the color patches. In order to assess the prediction quality, we used a visual metric: the color distance CIELAB $\Delta E_{94}$. As the final target of this spectral prediction model is to predict colors, this metric is more relevant than the spectral difference to estimate a perceptible difference between predicted and measured spectra. It also gives a well interpretable accuracy assessment scale, in particular with the just-noticeable color difference equal to 1 . It is obtained by converting the predicted and measured spectra into CIE-XYZ tristimulus values, calculated with a D65 illuminant and in respect to the $2^{\circ}$ standard observer, then by converting the CIE-XYZ values into CIELAB color coordinates using as a white reference 
the unprinted paper deduced from the reflectance spectrum of the paper illuminated with the D65 illuminant [27].

Let us first consider the printed paper. The IS-YNSN model was tested in reflectance mode with 125 patches. The patches were printed on different supports with the Canon PIXMA Pro9500 Mark II inkjet printer: Canon photo paper MP101 (matte), Canon photo PP201 (glossy), and APCO II paper (supercalendered, nonfluorescent). The two halftoning methods were tested: rotated cluster halftoning at 150 lines per inch and stochastic halftoning. The average $\Delta E_{94}$ value for the 125 patches and the $\Delta E_{94} 95$ quantiles are presented in Table 1. The optimal Yule-Nielsen factor $n$ is specified in the table. It was limited to 10 in the case where the optimal $n$ tends asymptotically to infinity. In transmittance mode, the APCO II paper is selected because its transmittance is higher than of the Canon photo papers. For each Canon paper, the model presented in Eq. (18) was tested for the $d i: 8^{\circ}$ and de $: 8^{\circ}$ geometries. As shown in Table 1 , the average $\Delta E_{94}$ value for the prediction for these sets in reflectance mode are less than 0.25 and are obtained for the optimal Yule-Nielsen factor minimizing the average $\Delta E_{94}$. For the Canon MP101, as well as for the Canon PP201, the predictions are seen to be accurate for both halftoning methods. Regarding the APCO II paper, the low values of average $\Delta E_{94}$ prove the model's accuracy in both reflectance and transmittance modes.

Regarding the printed transparencies, we tested the models presented in Section $\underline{5}$ with CMY halftones printed on $3 \mathrm{M}$ CG3460 inkjet transparency film with a Canon PIXMA Pro9500 Mark II inkjet printer. For each ink, the halftone dot screen was generated manually and printed independently of the other inks. This printing method in three independent passes enabled bypassing color corrections by the driver, thereby unknown modifications of the nominal surface coverages. In each test, the color differences between measured and predicted spectra, expressed in CIELAB $\Delta E_{94}$ values, are given in Table 2 . Several options were tested according to the different configurations proposed in Section 5. We can select either the reflectance mode based on a $d i: \overline{8}^{\circ}$ or $d e: 8^{\circ}$ geometry or transmittance mode based on a $0^{\circ}: 0^{\circ}$ geometry. We may also take into account the slight scattering of light by the inks $(K=1)$ or remove it $(K=0)$. The prediction accuracy for the different configurations are presented in Table 2. Let us explain how they were obtained. For a $d e: 8^{\circ}$ geometry, the spectral reflectance of the printed transparency is predicted thanks to Eq. (39). The predictions are very accurate because the average $\Delta \overline{E_{94}}$ value is lower than 0.3 for both halftoning methods. They are even improved by applying the Yule-Nielsen transform in Eq. (39). For the $d i: 8^{\circ}$ geometry, the spectral reflectance is predicted thanks to Eq. (43), with
$K=1$ (third row in Table 2 ) and $K=0$ (fourth row); poor predictions were obtained, probably because the effective surface coverages are deduced from transmittance measurements, while it is known that parameters calibrated in transmittance mode are generally not adapted to reflectance prediction (see [7]). However, rather good predictions are provided by the IS-YNSN model, calibrated as in transmittance mode but from measurements performed in reflectance mode (fifth row in Table 2). The spectral transmittance model expressed by Eq. (44) with $\theta_{0}=0$ was also tested without and with the Yule-Nielsen transform. Notice that the optimal $n$ value takes a lower value in transmittance mode than in reflectance mode, which was already noticed in [7] for halftones on paper. As for the reflectance mode, the Yule-Nielsen transform improves the prediction accuracy for both halftoning methods and the average $\Delta E_{94}$ value is less than 0.7 . The improvement is more pronounced with the stochastic halftoning because the ink dots are smaller and the Yule-Nielsen effect is consequently stronger than with rotated cluster halftoning. Finally, the very low $\Delta E_{94}$ differences between predictions and measurements show the good accuracy of the IS-YNSN model for either $d e: 8^{\circ}$ and $d i: 8^{\circ}$ reflectance geometries and the $0^{\circ}: 0^{\circ}$ transmittance geometry.

The model for printed transparency superposed with a printed paper, presented in Section 6 , was tested on $3 \mathrm{M}$ CG3460 inkjet transparency film and two types of paper: the Canon photo MP101 matte paper and the Canon photo PP201 glossy paper. Again, several options were tested: we selected either stochastic or rotated cluster halftoning, in either reflectance or transmittance mode, by taking into account light scattering by the inks $(K=1)$ or not $(K=0)$; in reflectance mode, we selected either the $d i: 8^{\circ}$ or the $d e$ : $8^{\circ}$ geometry. The color differences between predictions and measurements for the different options are presented in Table 3. As can be seen in Fig. 5, yellow ink significantly increases the reflectance in $d e: 8^{\circ}$ geometry in the spectral range $480-620 \mathrm{~nm}$. Therefore, by setting $K$ to 1 when only yellow ink is printed and to 0 when the halftone contains other inks, the global prediction is increased. As an example, for a solid yellow patch printed on a transparency superposed with an unprinted paper (MP101), the color difference between the measured and predicted spectra with $K=0$ is 1.76 , whereas it is 0.85 with $K=1$, as indicated in Fig. $\underline{7}$. Hence, for the testing color sets where yellow-only halftones are present, the prediction accuracy is better when the diffusion of light by the yellow ink is taken into account. Regarding the paper, better accuracy is achieved in $d e: 8^{\circ}$ geometry with the MP101 matte paper (average $\Delta E_{94}<1$ ) than with the PP201 glossy paper (average $\Delta E_{94}<1.26$ ). This is due to the fact that the matte

Table 1. Prediction Accuracy for Printed Papers

\begin{tabular}{|c|c|c|c|c|c|c|c|c|}
\hline \multirow[b]{2}{*}{ Paper } & \multirow[b]{2}{*}{$(R)$ or $(T)$ Mode } & \multirow[b]{2}{*}{ Geometry } & \multicolumn{3}{|c|}{ Rotated Cluster Halftoning } & \multicolumn{3}{|c|}{ Stochastic Halftoning } \\
\hline & & & $n$ & Av. $\Delta E_{94}{ }^{a}$ & $95-Q^{a}$ & $n$ & Av. $\Delta E_{94}{ }^{a}$ & $95-Q^{a}$ \\
\hline MP101 & $R$ & $d i: 8^{\circ}$ & 10 & 0.22 & 0.74 & 10 & 0.21 & 0.60 \\
\hline \multirow[t]{2}{*}{ PP201 } & $R$ & $d i: 8^{\circ}$ & 10 & 0.25 & 0.52 & 10 & 0.22 & 0.49 \\
\hline & $R$ & $d e: 8^{\circ}$ & 5 & 0.23 & 0.58 & 4 & 0.17 & 0.38 \\
\hline APCO & $R$ & $d i: 8^{\circ}$ & - & - & - & 4 & 0.22 & 0.56 \\
\hline
\end{tabular}

${ }^{a}$ Average color differences and 95 quantile over 125 tested halftone colors denoting the deviation between the measured spectra and the ones predicted with the IS-YNSN model for the indicated optimal $n$ value. 
Table 2. Prediction Accuracy for Printed Transparencies

\begin{tabular}{|c|c|c|c|c|c|c|c|c|}
\hline \multirow[b]{2}{*}{ Mode } & \multirow[b]{2}{*}{ Geometry } & \multirow[b]{2}{*}{ Model } & \multicolumn{3}{|c|}{ Rotated Cluster Halftoning } & \multicolumn{3}{|c|}{ Stochastic Halftoning } \\
\hline & & & $n$ & Av. $\Delta E_{94}{ }^{a}$ & $95-Q^{a}$ & $n$ & Av. $\Delta E_{94}{ }^{a}$ & $95-Q^{a}$ \\
\hline \multirow[t]{5}{*}{$R$} & de $: 8^{\circ}$ & IS-SN $^{b}$ & - & 0.28 & 0.82 & - & 0.29 & 0.99 \\
\hline & de $: 8^{\circ}$ & IS-YNSN & 10 & 0.25 & 0.70 & 10 & 0.24 & 0.84 \\
\hline & $d i: 8^{\circ}$ & Eq. (노) $K=1$ & - & 2.07 & 2.57 & - & 1.99 & 2.60 \\
\hline & $d i: 8^{\circ}$ & Eq. (푸) $K=0$ & - & 2.02 & 2.53 & - & 1.90 & 2.48 \\
\hline & $d i: 8^{\circ}$ & IS-YNSN & 4 & 0.16 & 0.41 & 2 & 0.15 & 0.48 \\
\hline \multirow[t]{2}{*}{$T$} & $0^{\circ}: 0^{\circ}$ & IS-SN $^{b}$ & - & 0.70 & 1.59 & - & 0.54 & 1.27 \\
\hline & $0^{\circ}: 0^{\circ}$ & IS-YNSN & 2 & 0.63 & 1.64 & 2 & 0.34 & 0.87 \\
\hline
\end{tabular}

${ }^{a}$ Average color differences and 95 quantile over 125 tested halftone colors denoting the deviation between the measured spectra and the ones predicted with the IS-YNSN model for the indicated optimal $n$ value.

${ }^{b}$ Ink-spreading enhanced spectral Neugebauer model, equivalent to the IS-YNSN model with $n=1$ (or equivalently $n$ ignored).

Table 3. Average Color Differences Denoting the Deviation between Measured and Predicted Spectral Reflectances

\begin{tabular}{|c|c|c|c|c|c|c|c|}
\hline$T+P$ Support & $(R)$ or $(T)$ Mode & Geom. & Halftoning Method & \multicolumn{2}{|c|}{$K=0^{a}$} & \multicolumn{2}{|c|}{$K=1^{a}$} \\
\hline CG3460 + MP101 & $R$ & $d i: 8^{\circ}$ & Stochastic & 0.83 & 1.51 & 0.83 & 1.51 \\
\hline \multirow[t]{2}{*}{ CG3460 + PP201 } & $R$ & $d i: 8^{\circ}$ & Stochastic & 0.92 & 2.02 & 0.89 & 1.77 \\
\hline & & & Rot. cluster & 1.16 & 1.96 & 1.13 & 1.85 \\
\hline CG3460 + MP101 & $R$ & $d e: 8^{\circ}$ & Stochastic & 1.01 & 1.92 & 1.01 & 1.92 \\
\hline CG3460 + PP201 & & & Rot. cluster & 1.26 & 3.01 & 1.21 & 2.59 \\
\hline CG3460 + APCO II & $T$ & $d: 0^{\circ}$ & Stochastic & 0.58 & 1.04 & 0.64 & 1.12 \\
\hline
\end{tabular}

${ }^{a} K=1$ is applied for colors containing only yellow ink, and $K=0$ is applied for the other colors (those containing cyan and magenta inks).

${ }^{b}$ Average color differences and 95 quantile over 125 tested halftone colors denoting the deviation between the measured spectra and the ones predicted with the IS-YNSN model for the indicated optimal $n$ value.

paper satisfies more closely the explicit assumption of Lambertian paper we made when developing the model. With the glossy paper, the specularly reflected light involved in the multiple reflection process is not well calibrated from measurements based on the $d e: 8^{\circ}$ geometry. Regarding the halftoning methods, the prediction accuracy seems to be as good for rotated cluster screening as for stochastic screening. We can therefore conclude that the moiré effects are not troublesome for the predictions and that rough alignment is sufficient to prevent moiré effects. In transmittance mode, we tested several hundred patches printed with the Canon PIXMA Pro9500 Mark II on APCO II paper and on the 3M CG3460 transparency film. The good fit evidenced by the average $\Delta E_{94}$ value of 0.58 was obtained with $K=0$.

Figure 8 shows three examples of predicted and measured spectra corresponding to different accuracy levels of the model. They come from three different samples where the surface coverages of the cyan, magenta, and yellow inks on the transparency and on the paper are, respectively, $(c, m, y)=$ $(0.25,0.25,0.25)$ and $(0.50,0.75,0.25)$ for Sample $1,(0.75$, $0.25,0)$ and $(0.25,0,0.25)$ for Sample 2 , and $(0,0,0)$ and $(0,0.25,0.50)$ for Sample 3. Measurements were performed with the $d e: 8^{\circ}$ geometry. As indicated by the $\Delta E_{94}$ values specified for each sample in the figure, the prediction accuracy tends to increase when the colors are darker, i.e., when the amount of ink increases. Spectra of sample 1 illustrate the case where prediction slightly deviates from measurement in a small waveband (here in the short-wavelength domain) and yields high equivalent color deviation. The spectra of sample 2 correspond to the maximal deviation between prediction and measurement that were observed in our experiments. The $\Delta E_{94}$ value of 2.36 indicates that the color prediction is not good, but we may be satisfied by the fact that the maximum deviation is rather limited. Such a maximal $\Delta E_{94}$ value is often encountered when predicting the spectral reflectance of halftones on paper; in the present case, we use three models, one for the paper, one for the transparency, and one for their

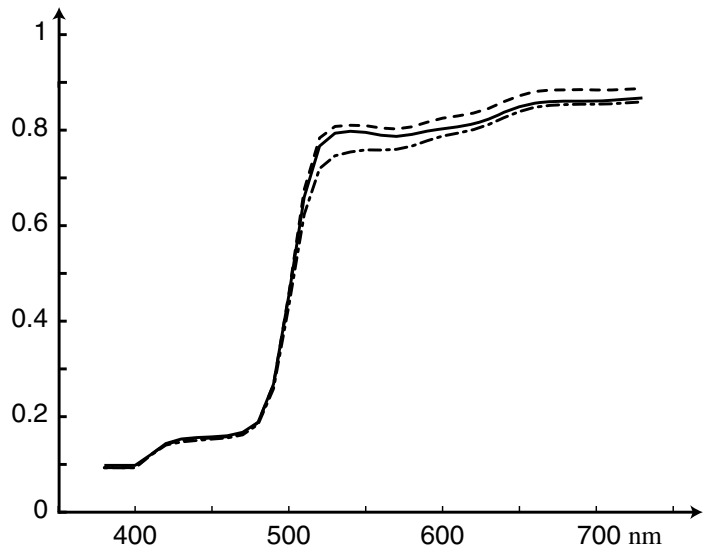

Fig. 7. Measured spectral reflectance of a solid yellow printed on the transparency superposed with the unprinted MP101 matte paper for a $d i: 8^{\circ}$ geometry (solid curve) and predicted spectra with $K=1$ (dashed curve) with $K=0$ (dashed-dotted curve). 


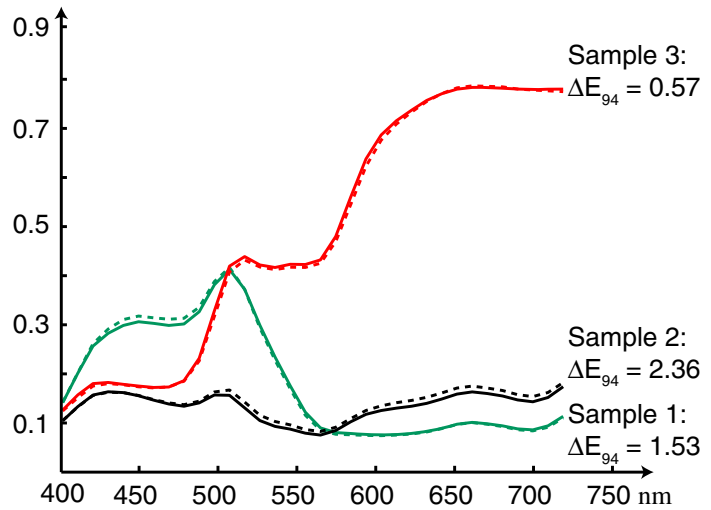

Fig. 8. (Color online) Measured (solid curve) and predicted (dashed curve) spectral reflectances at normal incidence of three superpositions of printed transparency and printed paper.

superposition, and the errors due to each model add to each other. For sample 3, predicted and measured spectra are almost superposed; they correspond to the lowest $\Delta E_{94}$ value obtained in our experiment.

\section{CONCLUSION}

In this paper, we have proposed the first reflectance and transmittance model for printed transparency. This model takes into account the lateral propagation of light between colorants (Neugebauer primaries) due to the multiple reflections of light within the transparency thanks to a Yule-Nielsen transform. It also enables taking into account the diffusing power of the inks in the eventuality that it affects noticeably the reflectance of the transparency. The model enables prediction of reflectance in and out of the specular direction. When the printed transparency is superposed with a printed paper, a second model predicts the reflectance or the transmittance of the stack from the spectral reflectance and transmittance of the transparency and the ones of the printed paper are predicted separately and used to determine their global reflectance and transmittance once they are superposed. This second model was established by following an original approach mixing the description of a multiple reflection process and the modelization of lateral light shifts in the halftone according to the Yule-Nielsen transform. This approach is probative according to the experimental test that we have carried out with several patches printed in inkjet at high screen frequencies. This approach remains valid as long as the light is not too much scattered by the inks printed on the transparency film; i.e., one can distinguish details of an object located at several meters when looking at it through the printed transparency, and as paper can be considered as a Lambertian reflector; i.e., no specular effect such as bronzing or metallic reflection is observed. An alternative approach would consist in modeling the multiple reflections between the paper bulk, the ink layers, the paper's interfaces, and the transparency's interfaces, while estimating the lateral shift of the light between the different colorants of the two halftones. This model would be much more complicated without ensuring that the accuracy would be improved. Therefore, the proposed model has the advantage of simplicity and performance. It could help to develop original applications in graphic arts where color images printed on transparency and paper are superposed.

\section{APPENDIX A: RELATIONSHIP BETWEEN REFLECTANCE AND REFLECTANCE FACTOR}

The reflectance factor is defined as the ratio of the flux reflected by the sample to the flux reflected by a perfect white diffuser measured in the same conditions.

The radiance $L_{D}$ directed toward the detector is defined as the ratio of the received element of flux $d^{2} \Phi_{d}$ to the product of the detector's surface $d s_{d}$ and solid angle $d \Omega_{d}$ :

$$
L_{D}=\frac{d^{2} \Phi_{d}}{d s_{d} d \Omega_{d}} .
$$

According to the transfer volume conservation principle, one has

$$
d s_{d} d \Omega_{d}=d s d \omega \cos \theta_{d},
$$

where $d s$ is the element area of the sample, $d \omega=d s_{d} / x^{2}$ is the solid angle based on the sample and subtended by the detector with $x$ the distance between the detector and the sample, and $\theta_{d}$ is the angle formed by the normal of the sample with the normal of the detector.

As the incident element of flux $d \Phi_{i}$ to the sample is related to the irradiance of the sample with $d \Phi_{i}=E_{i} d s$, the ratio between the received and the incident fluxes, using Eqs. (A1) and (A2) is

$$
\frac{d^{2} \Phi_{d}}{d \Phi_{i}}=\frac{L_{D} d s_{d} d \Omega_{d}}{E_{i} d s}=\frac{d s_{d} \cos \theta_{d}}{x^{2}} \frac{L_{D}}{E_{i}} .
$$

For a fixed orientation of the detector $\theta_{d}$, the ratio $L_{D} / E_{i}$ is proportional to a reflectance:

$$
R=\frac{d^{2} \Phi_{d}}{d \Phi_{i}}=\xi \frac{L_{D}}{E_{i}}
$$

where

$$
\xi=\frac{d s_{d} \cos \theta_{d}}{x^{2}}
$$

The factor $\xi$ only depends on the intrinsic parameters of the spectrophotometer (the surface of the detector $d s_{d}$, its orientation $\theta_{d}$ and its distance $x$ to the sample). For a perfect white diffuser, the ratio $L_{D} / E_{i}$ is equal to $1 / \pi$ according to the Lambert law. The reflectance $R_{\text {ref }}$ is therefore $\xi / \pi$.

Considering the reflectance of the sample, the reflectance factor $\hat{R}$ is expressed by

$$
\hat{R}=\frac{R}{R_{\text {ref }}}=\pi \frac{L_{D}}{E_{i}} .
$$

If the sample is a Lambertian reflector of reflectance $\rho$, the radiance reflected toward the detector is $L_{D}=\rho E_{i} / \pi$. The reflectance factor is therefore equal to the reflectance. 


\section{REFERENCES}

1. R. D. Hersch and F. Crété, "Improving the Yule-Nielsen modified spectral Neugebauer model by dot surface coverages depending on the ink superposition conditions," Proc. SPIE 5667, 434-447 (2005).

2. J. A. S. Viggiano, "Modeling the color of multi-colored halftones," in Proceedings of the Technical Association of the Graphic Arts (TAGA, 1990), pp. 44-62.

3. F. Clapper and J. Yule, "The effect of multiple internal reflections on the densities of halftones prints on paper," J. Opt. Soc. Am. 43, 600-603 (1953).

4. F. C. Williams and F. R. Clapper, "Multiple internal reflections in photographic color prints," J. Opt. Soc. Am. 43, 595-597 (1953).

5. M. Hébert and R. D. Hersch, "Reflectance and transmittance model for recto-verso halftone prints," J. Opt. Soc. Am. A 23, 2415-2432 (2006).

6. M. Hébert and R. D. Hersch, "Reflectance and transmittance model for recto-verso halftone prints: spectral predictions with multi-ink halftones," J. Opt. Soc. Am. A 26, 356-364 (2009).

7. M. Hébert and R. D. Hersch, "Yule-Nielsen based recto-verso color halftone transmittance prediction model," Appl. Opt 50, 519-525 (2011).

8. M. Hébert and R. D. Hersch, "Deducing ink-transmittance spectra from reflectance and transmittance measurements of prints," Proc. SPIE 6493, 649314 (2007).

9. M. Hébert, R. D. Hersch, and J. Becker, "Compositional reflectance and transmittance model for multilayer specimens," J. Opt. Soc. Am. A 24, 2628-2644 (2007)

10. M. Hébert, R. D. Hersch, and L. Simonot, "Spectral prediction model for piles of nonscattering sheets," J. Opt. Soc. Am. A 25, 2066-2077 (2008).

11. F. R. Ruckdeschel and O. G. Hauser, "Yule-Nielsen effect in printing: a physical analysis,” Appl. Opt. 17, 3376-3383 (1978).

12. P. Emmel, I. Amidror, V. Ostromoukhov, and R. D. Hersch, "Predicting the spectral behaviour of colour printers for transparent inks on transparent support," in Proceedings of IS\&T/SID 96, Color Imaging Conference (Society for Imaging Science, 1996), pp. 86-91.
13. J. McElvain, J. Miller, and E. Jin, "Spectral printer modeling for transparency media: toward high dynamic range scene reproduction," Proc. SPIE 7241, 72410U (2009).

14. I. Amidror, The Theory of the Moiré Phenomenon: Periodic Layers, 2nd ed. (Springer, 2009).

15. V. Ostromoukhov and R. D. Hersch, "Stochastic clustered-dot dithering," J. Electron. Imag. 8, 439-445 (1999).

16. M. Qi, C. Yang, C. Tu, X. Meng, and Y. Sun, "A GPU-based algorithm for building stochastic clustered-dot screens," Adv. Vis. Comput. 4841, 98-105 (2007).

17. J. Stover, Optical Scattering: Measurement and Analysis (SPIE Press, 1995).

18. F. Nicodemus, J. Richmond, J. Hsia, I. Ginsberg, and T. Limperis, Geometrical Considerations and Nomenclature for Reflectance (U.S. Dept. of Commerce, 1977).

19. M. Born, E. Wolf, and A. Bhatia, Principles of Optics: Electromagnetic Theory of Propagation, Interference and Diffraction of Light (Cambridge University, 1999).

20. CIE, Colorimetry CIE Technical Report, 3rd ed. (CIE, 1998).

21. H.-H. Perkampus, Encyclopedia of Spectroscopy (VCH, 1995).

22. L. Simonot, M. Hébert, and R. D. Hersch, "Extension of the Williams-Clapper model to stacked nondiffusing colored coatings with different refractive indices," J. Opt. Soc. Am. A 23, 1432-1441 (2006).

23. I. Amidror and R. D. Hersch, "Neugebauer and Demichel: dependence and independence in $n$-screen superpositions for colour printing," Color Res. Appl. 25, 267-277 (2000).

24. J. A. C. Yule and W. J. Nielsen, "The penetration of light into paper and its effect on halftone reproduction," in Proceedings of the Technical Association of the Graphic Arts, Vol. 3 (TAGA, 1951), pp. 65-76.

25. T. Bugnon and R. D. Hersch, "Constrained acquisition of ink spreading curves from printed color images," IEEE Trans. Image Process. 20, 513-522 (2011).

26. A. Glassner, Principles of Digital Image Synthesis, Vol. 2 (Kaufmann, 1995).

27. G. Sharma, Digital Color Imaging Handbook (CRC Press, 2003), pp. $30-36$. 